\title{
Coincidence Doppler Broadening of Positron Annihilation Radiation for Detection of Helium in Irradiated $\mathrm{Ni}$ and $\mathrm{Cu}$
}

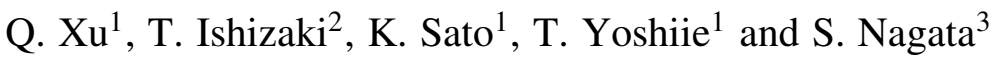 \\ ${ }^{1}$ Research Reactor Institute, Kyoto University, Sennan-gun, Osaka 590-0494, Japan \\ ${ }^{2}$ Toyota Central R\&D Labs., Aichi-gun, Aichi 480-1192, Japan \\ ${ }^{3}$ Institiute for Materials Research, Tohoku University, Sendai 980-8577, Japan
}

\begin{abstract}
A new composition analysis method, namely, coincidence Doppler broadening (CDB) of positron annihilation radiation, was employed to detect $\mathrm{He}$ atoms in ion irradiated $\mathrm{Ni}$ and neutron irradiated $\mathrm{Cu}$. The results of positron lifetime and transmission electron microscopy (TEM) show that microvoids and voids were formed in ion-irradiated $\mathrm{Ni}$ and neutron-irradiated $\mathrm{Cu}$, respectively. The results of $\mathrm{CDB}$ measurements indicate that $\mathrm{He}$ atoms were present in the microvoids and voids, even in microvoids annealed at $1273 \mathrm{~K}$ in ion-irradiated Ni. Coincidence Doppler broadening measurement, which is a nondestructive technique for testing materials, is effective for detecting He atoms. [doi:10.2320/matertrans.47.2885]
\end{abstract}

(Received July 18, 2006; Accepted September 15, 2006; Published November 15, 2006)

Keywords: helium, microvoids, voids, positron annihilation

\section{Introduction}

Interest in the behavior of $\mathrm{He}$ in solids has increased with advances in nuclear power technology, in particular, fusion reactor technology. He atoms can be generated in materials by the nuclear reaction of $(\mathrm{n}, \alpha)$. The production of He atoms induced by nuclear reactions increases with increasing neutron energy since the cross section of the $(n, \alpha)$ reaction generally increases with neutron energy. In addition, $\mathrm{He}$ atoms are introduced directly in plasma-facing materials by the He plasma in fusion reactors. These $\mathrm{He}$ atoms produce damage not only on the surface of materials, such as erosion, sputtering and blistering, but also in the bulk because $\mathrm{He}$ easily diffuses deep into the specimens. $\left.{ }^{1,2}\right) \mathrm{He}$ atoms are insoluble in most metals and alloys, ${ }^{3)}$ and the presence of $\mathrm{He}$ atoms in metals may have a great impact on the microstructure. He atoms promote the growth of cavities, ${ }^{4,5)}$ dislocation loops ${ }^{6,7)}$ and induce void swelling. These microstructural changes lead to the degradation of mechanical properties.

Thermal desorption spectrometry (TDS) is commonly used to detect $\mathrm{He}$ in materials. TDS detects He atoms exiting the sample. However, the TDS method has two disadvantages: i) the sample must be heated during the measurement, and ii) TDS is unable to provide information on sites trapping He.

Positron annihilation spectroscopy is a powerful tool for detecting vacancy-type defects in condensed matter. ${ }^{8)}$ Doppler broadening of positron annihilation radiation is a nondestructive technique for testing defect clusters. In the dominant decay mode of a thermal positron and electron, two gamma rays are emitted. In the laboratory frame, the energy of two photons emitted by the annihilation of a positron and an electron is different. The difference in photon energy is proportional to the longitudinal component of the electronpositron momentum in the direction of gamma emission. Measurement of photon energies yields information about the momentum distribution of core electrons. Thus, Doppler broadening measurements can provide useful information about the distribution of elements around annihilation sites.
Recently, Doppler broadening measurements have been improved by a two-Ge-detector coincidence system, which decreased the background of high momentum contributions by about two or three orders of magnitude compared with traditional measurements using a single Ge-detector. ${ }^{9)}$ Coincidence Doppler broadening (CDB) has been used widely to detect precipitates in alloys, ${ }^{10,11)}$ however, it has not yet been used to detect gaseous atoms. The main objective of the present work was to develop a new nondestructive technique for detecting $\mathrm{He}$ atoms in metals and alloys using CDB.

\section{Experimental Procedure}

In the case of ion irradiation, well annealed high-purity (99.99\%) Ni supplied by Johnson \& Matthey Chemicals Ltd. was irradiated with $3.3-\mathrm{MeV} \mathrm{He}$ ions at $423 \mathrm{~K}$ using an accelerator in the Institute for Materials Research, Tohoku University, at an irradiation dose of $9.6 \times 10^{15} \mathrm{He}$ ions $/ \mathrm{cm}^{2}$. The damage and the concentration of $\mathrm{He}$ at the peak region (about $6 \mu \mathrm{m}$ from the specimen surface) were $0.3 \mathrm{dpa}$ (displacements per atom) and $300 \mathrm{appm}$ based on calculations using TRIM code, ${ }^{12)}$ where the threshold displacement energy of $\mathrm{Ni}$ was assumed to be $24 \mathrm{eV}$. The peak width was $1 \mu \mathrm{m}$. The isochronal annealing experiments for irradiated $\mathrm{Ni}$ were carried out for $1 \mathrm{~h}$ at increments of $50 \mathrm{~K}$ from the irradiation temperature. To compare the formation of microvoids produced by He ion irradiation and neutron irradiation, where He production can be neglected, well annealed Ni was irradiated at the Kyoto University Reactor (KUR) ${ }^{13)}$ at $573 \mathrm{~K}$ to $4.4 \times 10^{-3} \mathrm{dpa}$.

Natural $\mathrm{Cu}$ is composed of $69.1 \mathrm{at} \%{ }^{63} \mathrm{Cu}$ and 30.9 at\% ${ }^{65} \mathrm{Cu}$. The (n, $\left.\alpha\right)$ and $(\mathrm{n}, \mathrm{p})$ reaction typically produces $\mathrm{He}$ and $\mathrm{H}$ in $\mathrm{Cu}$ during neutron irradiation. In order to avoid the effects of $\mathrm{H}$, the $\mathrm{Cu}$ isotope ${ }^{63} \mathrm{Cu}$, which was prepared by reductive reaction of ${ }^{63} \mathrm{CuO}$, was used in the neutron irradiation since $\mathrm{He}$ is produced only from ${ }^{63} \mathrm{Cu}$ by a $(\mathrm{n}, \alpha)$ reaction. The irradiation was conducted in a Fast Flux Test Facility (FFTF) reactor using the Materials Open Test 
Assembly (MOTA) below a core canister during its cycle 12 operation. The irradiation temperature and doses were $646 \mathrm{~K}$ and $7.3 \mathrm{dpa}$. The amount of $\mathrm{He}$ produced in ${ }^{63} \mathrm{Cu}$ was estimated to be 0.214 appm based on dosimetry measurements and calculations. ${ }^{14)}$ As in the case of $\mathrm{Ni}$, to compare the formation of microvoids produced by $\mathrm{He}$ ion irradiation and neutron irradiation, where He production can be neglected, well annealed $\mathrm{Cu}$ was irradiated at the KUR at $573 \mathrm{~K}$ to $2.8 \times 10^{-3} \mathrm{dpa}$.

Positron lifetime and coincidence Doppler broadening (CDB) were measured at room temperature. The positron lifetime spectrometer had a time resolution of $190 \mathrm{ps}$ (full width at half maximum), and each spectrum was accumulated to a total of $1.0 \times 10^{6}$ counts. To discriminate between bulk and defect components, after subtracting the source and background components, the lifetime spectrum $L(t)$ was decomposed into two components using the programs Resolution and Positronfit: ${ }^{15)}$

$$
L(t)=\left(I_{1} / \tau_{1}\right) \exp \left(-t / \tau_{1}\right)+\left(I_{2} / \tau_{2}\right) \exp \left(-t / \tau_{2}\right)
$$

where, $\tau_{i}$ are the lifetimes and $I_{i}$ are the intensities. The long lifetime $\tau_{2}$ comes from vacancies and vacancy clusters, if any, and the short lifetime $\tau_{1}$ results from the positron lifetime of free electrons and other defects, such as dislocations.

The average positron lifetime $\tau_{m}$ is defined as:

$$
\tau_{m}=I_{1} \tau_{1}+I_{2} \tau_{2} .
$$

Doppler-broadening spectra were accumulated to a total of $2.0 \times 10^{7}$ counts. The energy resolution was $1.4 \mathrm{keV}$ at $511 \mathrm{keV}$.

\section{Results and Discussion}

After irradiation to $9.6 \times 10^{15} \mathrm{He}$ ions $/ \mathrm{cm}^{2}$ in $\mathrm{Ni}$, the long lifetime $\tau_{2}$ was $278.8 \mathrm{ps}$ with an intensity of $13.6 \%$, which corresponded to a cluster of four vacancies $\mathrm{V}_{4} \cdot{ }^{16)}$ Figure 1 shows the lifetimes and intensities of long lifetime $\tau_{2}$ during annealing irradiated $\mathrm{Ni}$ to $1273 \mathrm{~K}$. The intensity of long lifetime almost did not change up to $723 \mathrm{~K}$, and then decreased with increasing annealing temperature. Meanwhile, the long lifetime decreased slightly during annealing from 623 to $823 \mathrm{~K}$, and then increased at $873 \mathrm{~K}$. The long lifetime increased to about $350 \mathrm{ps}$ at $1123 \mathrm{~K}$, which corresponded to a $\mathrm{V}_{10}$. These results indicate that the microvoids are stable at temperatures below $623 \mathrm{~K}$. It is believed that the decrease and increase in long lifetime were caused by microvoids absorbing the interstitials produced by dissociation or movement of tiny interstitial clusters and the migration of tiny microvoids such as $\mathrm{V}_{2}$ and $\mathrm{V}_{3}$, respectively. In addition, the lifetime spectra could not be decomposed into three components. In other words, positronium formation was not observed in lifetime measurements.

Figure 2 shows typical ratio curves of He-ion-irradiated $\mathrm{Ni}$ and after annealing at $1273 \mathrm{~K}$ to unirradiated $\mathrm{Ni}$. In order to identify $\mathrm{He}$, the figure also shows the ratio curve of neutronirradiated $\mathrm{Ni}$, where there was no He production, relative to $\mathrm{Ni}$. After irradiation, the ratio curves were higher than 1 in the low momentum region because more positrons were annihilated by valence electrons at vacancies in the irradiated
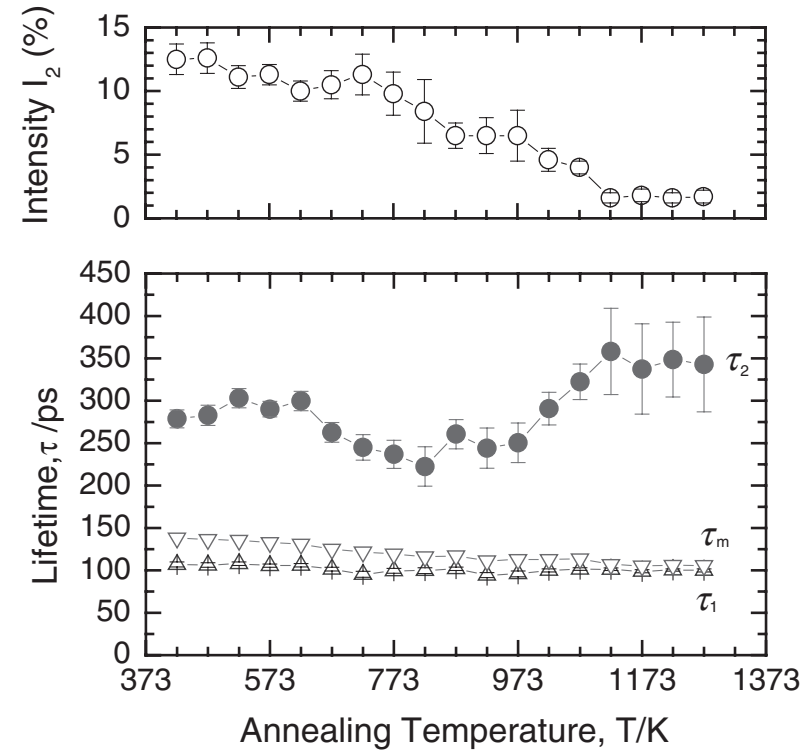

Fig. 1 Positron lifetimes and intensities of long lifetime in He-ionirradiated Ni during isochronal annealing experiments.

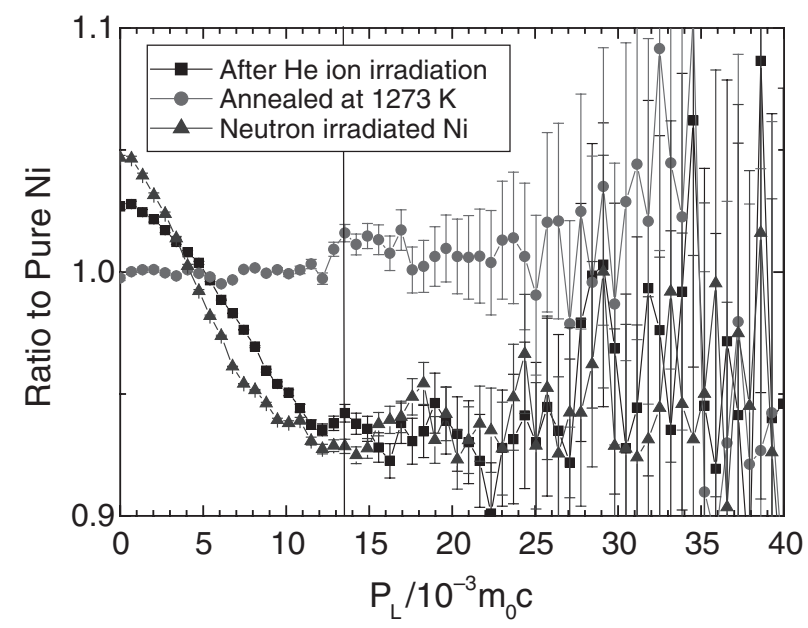

Fig. 2 Typical CDB ratio curves for He-ion-irradiated $\mathrm{Ni}$ after annealing at $1273 \mathrm{~K}$ and neutron-irradiated Ni relative to unirradiated $\mathrm{Ni}$.

samples. In addition, the ratio curve of He-ion-irradiated $\mathrm{Ni}$, especially after annealing at $1273 \mathrm{~K}$, shows a peak at about $13.5 \times 10^{-3} \mathrm{~m}_{0} \mathrm{c}$, where $\mathrm{m}_{0}$ is the electron rest mass, and $\mathrm{c}$ is the velocity of light. However, there was no such peak in neutron-irradiated $\mathrm{Ni}$ at $13.5 \times 10^{-3} \mathrm{~m}_{0} \mathrm{c}$. In the present study, we introduced two parameters, namely $\mathrm{S}$ and $\mathrm{W}$, defined as the ratio of the low-momentum $\left(\left|P_{\mathrm{L}}\right|<4 \times\right.$ $\left.10^{-3} \mathrm{~m}_{0} \mathrm{c}\right)$ and high-momentum $\left(11.5 \times 10^{-3} \mathrm{~m}_{0} \mathrm{c}<\right.$ $\left|P_{\mathrm{L}}\right|<15.5 \times 10^{-3} \mathrm{~m}_{0} \mathrm{c}$ ) regions in the Doppler broadening spectrum to the total region, respectively. $S$ represents the smaller Doppler shift resulting from the annihilation at the valence electrons of $\mathrm{Ni}$. In the same materials, the increase in $S$ relative to a well-annealed sample comes from the annihilation at vacancy-type defects. W comes from the annihilation at electrons of $\mathrm{He}$, which is used to estimate the number of $\mathrm{He}$ atoms around positrons when they are annihilated. Figure 3 shows the results of $\mathrm{S}$ and $\mathrm{W}$ parameter correlations for $\mathrm{He}$-ion-irradiated $\mathrm{Ni}$ during the annealing 


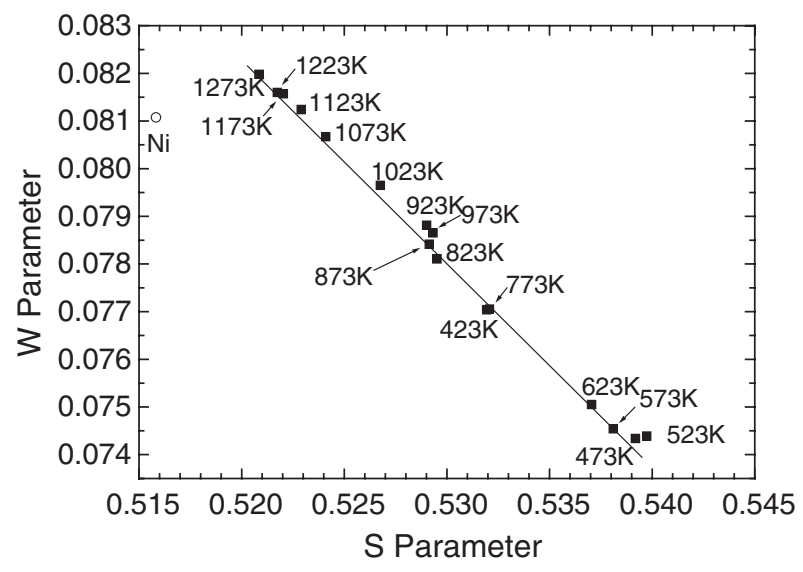

Fig. 3 Annealing temperature dependence of the S-W plot.

experiment. S increased and W decreased from 423 to $523 \mathrm{~K}$. The irradiation-induced vacancies became mobile at temperatures above $423 \mathrm{~K}$, and microvoids nucleated and grew though they could not be observed in the lifetime measurement. Then, $\mathrm{S}$ decreased and $\mathrm{W}$ increased with increasing annealing temperature. On the basis of the lifetime results described above, the decrease in S corresponded to annihilation with interstitials at relatively low temperatures $(<873 \mathrm{~K})$ and to a decrease in the microvoid concentration during growth of microvoids at temperatures above $873 \mathrm{~K}$. The $(\mathrm{S}, \mathrm{W})$ points of annealed Ni were almost aligned on the same line segment, which indicated that the variation in $S$ and $\mathrm{W}$ was only caused by the changes in microvoid size. In addition, with increasing annealing temperature, $\mathrm{S}$ closed to the value of unirradiated $\mathrm{Ni}$, but $\mathrm{W}$ remained higher than that of unirradiated $\mathrm{Ni}$. This means that $\mathrm{He}$ atoms were present even in microvoids annealed at high temperatures (1273 K). As the affinity of positrons is higher for microvoids than for $\mathrm{He}$ atoms, the positrons trapped at microvoids were annihilated with the electrons in $\mathrm{He}$, i.e., $\mathrm{He}$ was present in the microvoids.

In order to identify the He peak shown in Fig. 2, CDB measurements were carried out in neutron-irradiated isotope ${ }^{63} \mathrm{Cu}$, where $\mathrm{He}$ was produced homogeneously in the matrix by the nuclear reaction of $(\mathrm{n}, \alpha)$. Figure 4 shows ratio curves of neutron-irradiated ${ }^{63} \mathrm{Cu}$ relative to unirradiated $\mathrm{Cu}$. The figure also shows the ratio curve of low dose neutronirradiated natural $\mathrm{Cu}$, where the neutron dose was $0.0028 \mathrm{dpa}$ and $\mathrm{He}$ production could be neglected, relative to $\mathrm{Cu}$. Low dose neutron-irradiated $\mathrm{Cu}$ was selected because $\mathrm{S}$ is almost the same as that in neutron-irradiated ${ }^{63} \mathrm{Cu}$, which allows easy comparison. Though not prominent, there was a peak at about $13.5 \times 10^{-3} \mathrm{~m}_{0} \mathrm{c}$ in neutron-irradiated ${ }^{63} \mathrm{Cu}$. However, there was no peak in low dose irradiated $\mathrm{Cu}$. The low peak was due to the low concentration of He produced by the nuclear reaction, which was estimated to be 0.214 appm as described above. There were peaks at the same positions in He-ion-irradiated $\mathrm{Ni}$ and neutron-irradiated ${ }^{63} \mathrm{Cu}$. This indicates that the momentum distribution of $\mathrm{He}$ in metals is not influenced by the metallic element. The TEM observation shows that large voids with an average diameter of $100 \mathrm{~nm}$ were formed in neutron-irradiated ${ }^{63} \mathrm{Cu}$. The long lifetime $\tau_{2}$

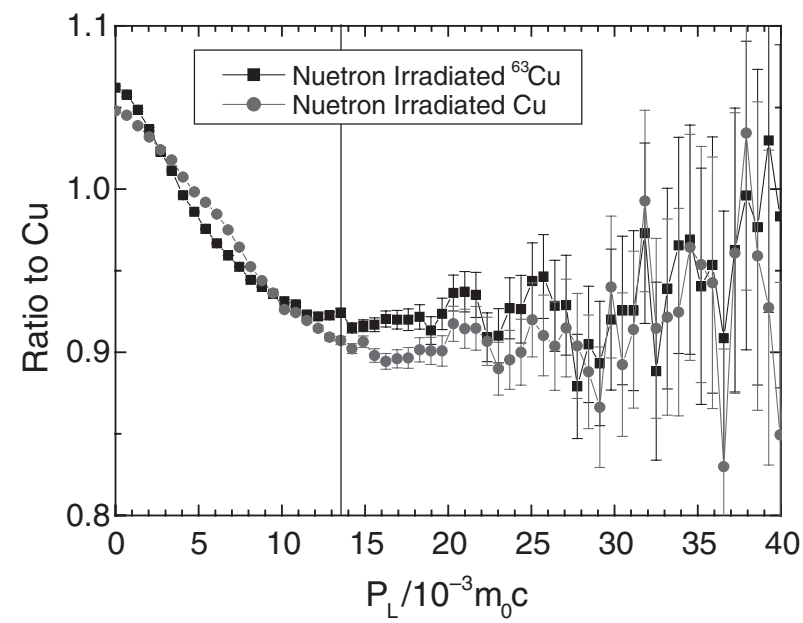

Fig. 4 Typical CDB ratio curves for neutron-irradiated ${ }^{63} \mathrm{Cu}$ and natural $\mathrm{Cu}$ relative to unirradiated $\mathrm{Cu}$.

was 439.2 ps with an intensity of $11.3 \%$. The long lifetime corresponded to the lifetime of the void surface since the voids were large. However, no formation of positronium was observed.

\section{Conclusion}

The present study employed a new detection method involving $\mathrm{CDB}$ to detect He in metals. He could be detected by $\mathrm{CDB}$ measurements even at low concentrations. The microvoids containing $\mathrm{He}$ were stable at the high temperature of $1273 \mathrm{~K}$. No positronium formation was detected in microvoids containing He.

\section{REFERENCES}

1) Q. Xu, N. Yoshida and T. Yoshiie: Mater. Trans. 46 (2005) 1255.

2) Q. Xu, N. Yoshida and T. Yoshiie: J. Nucl. Mater. (2006) in press.

3) M. D'Olieslaeger, G. Knuyt, L. De Schepper and L. M. Stals: Fundamental Aspects in Inert Gas in Solids Ed, by S. E. Donnelly and J. H. Evans, (Plenum, 27, 1991).

4) J. B. Conndon and T. Shober: J. Nucl. Mater. 207 (1993) 1.

5) K. Farrell: Rad. Eff. 53 (1980) 175.

6) K. Ono, T. Kino, S. Furuno, K. Hojou, K. Izui, K. Mizuno and K. Ito: J. Nucl. Mater. 179-181 (1991) 978.

7) K. Niwase, T. Ezawa, T. Tanabe, M. Kiritani and F. E. Fujita: J. Nucl. Mater. 203 (1993) 56.

8) A. Dupasquier and A. P. Mills, Jr.: Positron Spectroscopy of Solids (IOS Press, Amsterdam, 1995).

9) P. Asoka-Kumar, M. Alatalo, V. J. Ghosh, A. C. Kruseman, B. Nielsen and K. G. Lynn: Phys. Rev. Lett. 77 (1996) 2097.

10) Q. Xu, T. Yoshiie and K. Sato: Phys. Rev. B 73 (2006) 134115.

11) Q. Xu and T. Yoshiie: Mater. Sci. Forum 445-446 (2004) 216.

12) J. P. Biersack and L. G. Haggmark: Nucl. Instrum. Meth. 174 (1980) 257.

13) T. Yoshiie, Y. Hayashi, S. Yanagita, Q. Xu, Y. Satoh, H. Tsujimoto, T. Kozuka, K. Kamae, K. Mishima, S. Shiroya, K. Kobayashi, M. Utsuro and Y. Fujita: Nucl. Instrum. Methods, Phys. Res. A 498 (2003) 522.

14) L. R. Greenwood: Fusion Reactor Mater. Semiann. Prog. Rep. DOE/ ER-0313/18, 27 (1995).

15) P. Kirkegaard, M. Eldrup, O. E. Morgensen and N. J. Pedersen: Comput. Phys. Commun. 23 (1981) 307.

16) M. J. Puska and R. M. Nieminen: J. Phys. F: Met. Phys. 13 (1983) 333. 